\title{
Technologies Lead to Adaptability and Lifelong Engagement with Culture Throughout the Cloud
}

\author{
Silvia de los Rios Perez, Maria Fernanda Cabrera-Umpierrez, \\ Maria Teresa Arredondo, Shanshan Jiang, Jacqueline Floch, \\ and Maria Eugenia Beltran
}

\begin{abstract}
Cultural heritage represents one of the most important drivers for personal development, social cohesion and economic growth in Europe. Although the general population is aware of this fact, cultural heritage is still underexplored and cultural activities are not incorporated into citizens' lifestyle. Technology offers a potential to increase awareness about cultural offerings and create a public engagement with Culture. The current digital solutions adopted by cultural heritage institutions fail to achieve a lifelong engagement, and thus do not support institutions in increasing the number of visitors and retaining them. This chapter illustrates how cloud-based technologies can be exploited to increase a cultural lifelong engagement. We use the cloud to support technologies that enable adaptive and personalised cultural experiences according to individuals' interests, co-creation of cultural heritage experiences, and active user contribution to social storytelling. The work presented here is a result of the European co-funded project TAG CLOUD.
\end{abstract}

S. de los Rios Perez • M.F. Cabrera-Umpierrez $(\square) \bullet$ M.T. Arredondo

Universidad Politecnica de Madrid, Madrid, Spain

e-mail: srios@1st.tfo.upm.es; chiqui@1st.tfo.upm.es; mta@1st.tfo.upm.es

S. Jiang • J. Floch

SINTEF ICT, Trondheim, Norway

e-mail: shanshan.jiang@sintef.no; jacqueline.floch@sintef.no

M.E. Beltran

INMARK Estudios y Estrategias, Madrid, Spain

e-mail: xenia.beltran@grupoinmark.com 


\section{Cultural Heritage and Digital Technology: Paradigm or Reality?}

Cultural heritage is an important asset and a strategic resource for social, economic and environmental development in Europe (European Commission-Press Release 2014). As stated by Jose Manuel Barroso, president of the European Commission between 2004 and 2014, it is one of the most important drivers for personal development, social cohesion and economic growth (European CommissionPress Release 2007). However, cultural heritage is still underexplored. The general public usually incorporates few cultural activities in their life style. According to the data from the Special Eurobarometer 399 on 'Cultural access and participation' (2013) the engagement with cultural heritage became depressed after the beginning of the financial and economic crisis in 2007. Figures suggest that: about half of the European population does not visit any historical monument or site; only $37 \%$ of the population has visited a museum or gallery; and involvement in other cultural activities such as attending a concert or visiting a public library is less than $35 \%$.

Currently, museums and cultural heritage institutions have invested and are investing significant resources to introduce cultural heritage in the digital era. Curators and professionals in the heritage sector strive to attract, engage and retain visitors to heritage institutions (i.e. libraries, museums, archives and historical societies) using a range of digital technologies from relatively cheap interactive websites to expensive on-site 3D visualisations. Despite the usage of these technologies, no significant increase in the number of visitors has been reached. Few cultural institutions have incorporated innovative personalised digital approaches as part of their solutions, and few take into account cultural trends to engage visitors. Having said this, some cultural institutions have already integrate web 2.0 tools to enable users to share their experiences and create user generated material, in order to enhance their web presence and establish long term relationships with people (Ardissono et al. 2012). However, a wide part of European institutions do not yet incorporate technology for more than basic purposes, mainly focused in promotion (Saldaña et al. 2013), and this represents a major drawback where digital content about cultural objects is still 'centrally' produced by experts (e.g. curators, historians and archaeologists) rather than being co-created together with visitors. When personalisation is supported, it is also centrally defined and based on general views about the background and preferences of the general population. This means that experiences are adapted to common interests of clusters of similar users or stereotypes, but not to individuals with particular interests and preferences. Digital technologies support the creation of new ways of interaction between cultural heritage institutions and their visitors. They facilitate the move from consumer to active creator of personal cultural experiences. This chapter shows how the European co-funded project TAG CLOUD has confronted these challenges. With a multidisciplinary consortium formed by partners from five different European countries TAG CLOUD proposes to create lifelong cultural experiences by using cloud-based solutions that support adaptive and personalised cultural experiences according to individuals' interests, 
co-creation of cultural heritage experiences, and active user contribution to social storytelling.

The increase of the available information about cultural heritage on digital media, such as the web and social media, offers a potential to promote cultural heritage and develop new ways to participate in culture. The number of digital objects available in open data platforms has increased significantly. For instance, Europeana (2015), the European database for cultural heritage, currently provides access to over 33 million digitised objects, having reached 30 million objects in November 2013 (Report on the Implementation of Commission Recommendation 2011/711/EU). This means that, although around $82 \%$ of Europe's cultural collections (on average) are still not digitised, the trend is to increase these numbers (Borowiecki and Navarrete 2015). Critically this amount of information may cause a loss of perspective about what is important or interesting for each user and/or may overwhelm them. While metadata structures such as those implemented by OGD (Open Government Data), and Europeana, that model cultural data through the EDM (Europeana Data Model) are helping to standardise the process of digital collection, a big amount of the available cultural digital content is still represented in non-standardised manners, and/or lack most of the fields of the corresponding metadata schema. This represents a big barrier to the access, use and re-use the content. The information should therefore be firstly curated and stored in a standardised way that will enable its future manipulation, use and re-use, and identification of what is relevant for whom. In that way, it is possible to provide suitable personalised information to each user.

The public, in general, differs when enjoying a cultural experience and this experience is composed of physical, personal, social context and identity-related aspects (Falk 2009). However, some of these aspects evolve and change during the visit to the cultural institution and/or the life of the user, leading to a need for continuous adaptation. Therefore, engagement techniques are required that not solely support user-tailored and personalised interactions with digital cultural artefacts, but also can adapt to the changing needs of the visitors. We observe that curators and professionals in the heritage sector recognise that lifelong cultural experience is the best way to engage the public. In fact, personalisation and adaptation play a main role for making the current cultural heritage experience a lifelong one (Wilkening and Chung 2009). For this purpose, there exists the need to dynamically update user profiles, to analyse past experiences, to collect past and current evidences, to remove, to add and change users' preferences, to track the interest and trends of the users in order to become a life representation of themselves, and to provide dynamic personalisation of the cultural experiences according to their current interests, their past experiences and the context of the current experience.

Current digital solutions for cultural heritage initiatives do not provide adequate personalised experiences (Vassileva 2012). Digital technology offers a potential to provide a suitable one-size-fits-one personalisation, as each individual is unique and thus needs a unique solution. For example, in the last years, web applications for commercial purposes have widely adopted the social web as a source of reliable 
data for personalisation to increase their sales. Social media platforms offer different services, such as user models, and profiles of various entities such as people, companies and places. These social media profiles have associated information, such as name, location, and birthday that may be related to a person, company or place, as well as different relationships and interactions between people, such as: friendship, follower or followed, check-in, etc. Thus social media can provide a lot of information about the user in order to create a cultural user profile. His/her likes, interests, activities on the social web and also about his/her real life (e.g. check-ins into places, likes of music, film and place pages) are relevant. Also, information provided by his/her peers can be important for personalisation. Recently, cultural heritage institutions and curators have used different social media channels, such as Facebook and Twitter, to support the clustering of the users' community (Bernstein 2008). In addition to the acquisition of knowledge about the users, social media supports user participation and collaboration through virtual social interactions, and games. This can occur both in real time and in an asynchronous way. Social media also offers a new expression channel. The sharing of contents, such as videos, photographs and stories, can be exploited as a new source for unmediated heritage. But again, this approach, although relevant for cultural heritage sites, is still a step behind in providing a true individual experience.

In order to provide the proposed lifelong engagement and generate unique content for each user, current systems and solutions should evolve towards effective and adaptive cultural systems that aim to add value and new meaning to cultural digital artefacts and place users as active creators instead of mere consumers of cultural heritage. Cloud based systems offer a unique potential in this direction because they offer the processing of huge amounts of data that may come from different sources, and even at the same time; apply different treatments to the data in order to format it for the desired purposes; and offer a set of services suitable for each of the desired features. This is the reason that led TAG CLOUD to propose a cloud-based system to increase cultural lifelong engagement. The characteristics that this new generation of cloud-based cultural systems, such as the one developed by TAG CLOUD, offers can be summarised as follows:

- Exploration and discovery of cultural initiatives according to the users' likes, interests and preferences.

- Recommendation of experiences to new areas based on other users' cultural timelines.

- Co-creation of cultural heritage, as the process that both cultural institutions and users are involved in the generation of cultural contents and the forming of cultural experiences.

- Fusion between information from experts about artefacts and cultural heritage institutions (or mediated heritage, i.e. cultural heritage that is managed, held, curated, transmitted in or through cultural institutions) and cultural user-content from social media, also known as living media (or unmediated heritage, i.e. cultural heritage that is independently produced, transmitted, shared or exists 
without the management involvement or mediation of cultural agencies or institutions).

- The possibility to manage and process large quantities and growing digital contents and objects.

- Re-use of the curated digital cultural content in other contexts.

- The enabling of real-time geographical mapping to increase user experience.

- Feedback for cultural sites' curators and managers to create/detect 'hot spots' as well as create/improve demand-oriented content.

- Compatibility with standards in order to facilitate the future management, use and re-use of cultural digital content.

This chapter presents how cloud-based technologies allow an adaptive and personalised cultural experience by seamlessly incorporating cloud-based (non-sensitive) information about the habits, preferences and motives of individuals into the digital content of a cultural object (e.g. artefacts, buildings and sites), aiming to increase users' interest in cultural heritage. In this way, users are actively invited to participate in the assignment of the importance of a cultural artefact and they become participants in the creation of their own cultural experiences through the creation and sharing of information on social media. Our assumption is that this new relationship between individuals and cultural heritage has the potential to make users adopt cultural heritage as part of their life-style and to enable lifelong cultural experiences. Our work is part of the European co-funded project TAG CLOUD, which has developed several digital solutions as outcomes and tangible results to cope with its objectives; they include the COOLTURA Platform and App, and stedr App.

The COOLTURA Platform is a cloud-based, open data-oriented platform that enables scalable services, such as harvesting of cultural content, semantic enrichment, personalisation and contextual adaptation of cultural content. In addition, the platform supports the curation processes for digital cultural content and artefacts; and offers tools to map, build and increase the metadata structure of the harvested content towards the OGD metadata scheme in order to tackle the challenge of achieving standardisation of cultural content representation to facilitate its access, use and re-use. The COOLTURA App is an application developed for mobile devices that allows visitors and users to experiment with different types of interactions with cultural objects (e.g. augmented reality, interaction with physical objects such as totems), as well as recommend new experiences based on the earlier user behaviour. Stedr ${ }^{1}$ is a mobile application for social storytelling and for discovering, creating and sharing digital stories related to places. It provides a revisited storytelling approach that fuses traditional digital storytelling with social media as a way for the co-creation of cultural heritage.

In order to give a brief overview of how these initiatives are connected, it is worth mentioning that the COOLTURA Platform provides a set of cloud services

\footnotetext{
${ }^{1}$ The name of the application stems from the Norwegian word sted (plural steder) for place.
} 
that can be connected to different clients (i.e. different types of cultural applications). This way, as a proof of concept, the COOLTURA App is the first application that accesses, uses and re-uses the cultural content processed in the COOLTURA Platform, and thus provides an individual personalised and adapted experience to the user. Later, new applications, such as stedr, can be connected as well to benefit of the services provided by the COOLTURA Platform.

Within the scope of the project, COOLTURA and stedr have been implemented, piloted and later deployed in three cultural sites located in very different environments, while managing very different forms of heritage:

- The Monumental Complex of the Alhambra and Generalife, in Granada, Spain: a monumental complex with indoor and outdoor spaces, which is situated in a medieval city on the foot of the Sierra Nevada Mountains.

- The Barber Institute of Fine Arts, in the West Midlands, United Kingdom: an indoor museum, which owns the Byzantine Coin Collection, the finest Byzantine collection worldwide housed in an Art Deco building in the heart of an international university campus and on the periphery of the land-locked cradle of Britain's industrial revolution.

- The County of South-Trøndelag (Sør-Trøndelag), in Norway: an open landscape with a rich heritage linked to seafaring, that is placed in mid-Norway and holds the third largest city in Norway, Trondheim, regional capital of Sør-Trøndelag.

\section{Engaging People with Cultural Heritage Through...}

\section{1 ...The Adaptation of Cultural Experiences}

To motivate an engagement with the general public about their cultural heritage requires their interest to participate in cultural experiences. Our approach to 'engage' users with cultural heritage and the community is in close relation, and complementary activity, with the personalisation mechanisms offered through adaptive experience. Digital solutions for the presentation of cultural offerings are traditionally based on a general view about the common background and preferences of the general population, or particular group of visitors targeted by the cultural institution. They fail to adapt to the diverse preferences of a heterogeneous public. This is the main problem that the set of digital solutions developed in TAG CLOUD are addressing, through the support for the adaptation of cultural experiences to each individual user.

TAG CLOUD exploits social media so that it can connect with, personalise and adapt the cultural experience; and also motivate the users and their peers to engage with their cultural environment. Thus, social media is used in a two-fold approach: (1) to gather information about the profile of each individual user for personalisation purposes, and, (2) to facilitate the active participation of the users and engage them to become co-creators of cultural heritage. Social media is used by and circulates among millions of people all over the world. It is used for creating 
and sharing content (i.e. comments and pictures) as a new way of expression. Some content is automatically generated once the user clicks a button (such as likes in Facebook) and some is provided by users and their peers (e.g. posts, comments and tweets). The interaction on a social media is mainly done through a variety of services to acquire or generate information from/to other peers. All of the information available on social media allows gathering a collective and rich source of data about the users, and offering a personal experience.

Social media is extensively used all over the world, with millions of active users involved. Taking into account impact and the large amount of information that can be retrieved from the profile of the user and activity on social media, there is a huge potential to personalise and adapt services and produce social engagement using cloud-based technologies in combination with social media. In addition, social media can provide a large amount of information about the user, both from his/her profile and from his/her activities and social interaction. However, a key issue is the privacy of each individual's data. In TAG CLOUD a privacy policy has been created in line with the EU laws and TAG CLOUD's stance on privacy. This policy is presented to the user when starting to use the application and is followed by TAG CLOUD at each stage.

All of this information about the user coming from social media enables a dynamic update of the user profile that serves to personalise the services. We provide personalised cultural experiences according to the users' likes and interests, and recommendations evolve according to their activities. In TAG CLOUD, the mobile COOLTURA App is the main entry point for the user to a cultural discovery adapted and provided by the COOLTURA Platform. COOLTURA uses a hybrid motivation methodology, which combines gamification techniques, intrinsic motivation and reciprocity, with the objective to motivate the users to participate in social networks to create, share and disseminate their cultural heritage. In addition, in order to achieve a better personalisation of the interaction with the cultural artefacts and better adaptation of the content, the user profile is continuously updated.

Using the COOLTURA App, the user receives recommendations about cultural offerings based on his/her personal profile. A personal profile includes interests provided by the user, interests extracted from social media, previous cultural experiences, the time spent on different offerings and feedback to these previous experiences (e.g. what the user liked). The recommendation system exploits content-based filtering, i.e. filtering according to categories, and collaborative filtering, i.e. filtering according to similarities with other users. In addition to interests, the system can also exploit user location in order to select among offerings in the vicinity of the user. Recommendations can be applied at different levels:

- At the cultural site level. The user is provided with an overview of relevant cultural sites.

- At the point of interest level. The user is provided with an overview of relevant places or objects in a cultural site. 

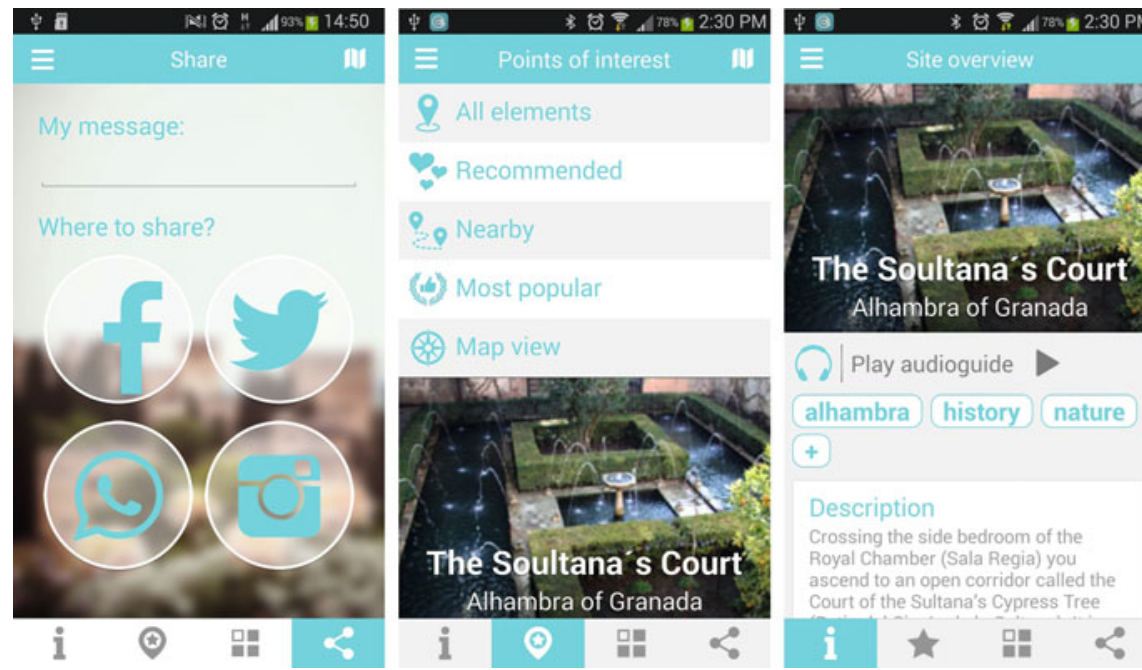

The Soultana s Court Alhambra of Granada
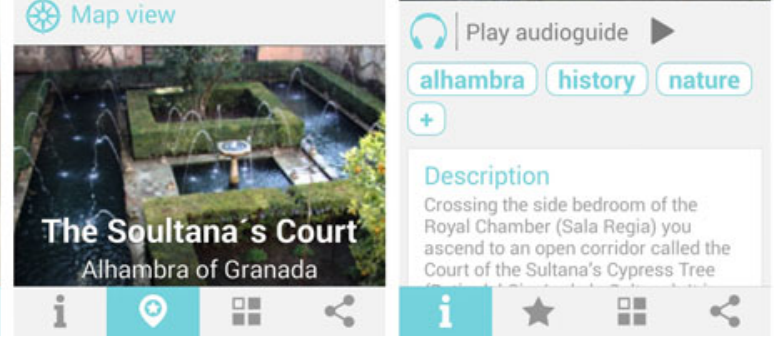

Fig. 1 COOLTURA screenshots. On the left, the screen to share in social media, in the middle, the different options to sort the points of interest, on the right, the description of a point of interest

- At the narrative level. The user is provided with different stories related to a point of interest.

- At the digital artefacts level. The user is provided with a list of relevant digital artefacts related to a point of interest.

Besides recommendation of cultural content, the COOLTURA App also supports different digital interaction modes (called activities in the App), such as augmented reality, storytelling and games. Currently, the user can receive recommendations regarding specific interaction modes, but is free to select among a set of digital interactions or switch between interactions. The COOLTURA App could be extended with application modules that support new digital interaction modes in the future. For instance, a new game could be added (Fig. 1).

Providing an adaptive cultural experience is a way to engage users. The personalised cultural experience through the COOLTURA App is not just a set of cultural offerings 'pushed' from the cultural institutions, but instead, is the result of a co-creation process where both cultural heritage institutions and visitors have shared their needs, requirements and insights. To reach adaptive cultural experiences, the cultural institutions or sites provide an architectural baseline (i.e. contents and interaction modes), and the users can dynamically generate their own experiences, by either (1) directly selecting the interaction modes or switching between interactions (i.e. activities), or (2) indirectly by receiving cultural recommendations from the COOLTURA Platform based on their user profile. In TAG CLOUD, the user profile is dynamically updated along with the user's experiences, evolving interests and preferences. By giving feedback or by 
experiencing an offer, the user influences recommendations. Therefore, personalisation provides a dynamic experience that continuously transforms. In addition, social media leveraged in COOLTURA App allows users to share their comments and personal experiences, assuming more active roles for participation like 'critics' and 'creators' (Simon 2010).

\section{$2.2 \quad$...Social Storytelling}

Stories drive people to feel. They broaden our knowledge. They make us reflect and change behaviour. Stories have long been used in cultural heritage institutions. There is no more special an experience than visiting a cultural site in the company of a guide who tells fascinating stories about the exhibits. When human guides are scarce resources, digital technology offers the chance to bring these experiences to a wider audience. An initial study done by TAG CLOUD shows that, indeed, people favour traditional cultural discovery approaches, such as storytelling and itineraries (Floch and Jiang, HCITOCH 2015). Therefore it was important to support storytelling in the COOLTURA Platform.

Similar to existing digital technology approaches, the COOLTURA Platform is combined with visual and spoken communication, and exploits different types of media such as audio, pictures or videos. Beyond presenting stories authored by cultural institutions, we provide the users with tools to contribute to storytelling. A participatory approach is an opportunity to enrich the portfolio of cultural stories provided by professionals and allow the visitor to connect with culture. There are often diverse ways to look at cultural artefacts, this means that there are also diverse ways to talk about them, and thus there is a potential to retain the attention of people with different interests. Further several treasures in our cultural heritage do not exist under the responsibility of specific cultural institutions, or in some cases few resources are available to document and present them, which makes it difficult to document history related to those artefacts. However, we still know that there are many cultural enthusiasts that are eager at documenting cultural heritage around them, e.g., members of local history associations.

There are many ways to tell a story. Advanced narratives that combine text, audio, pictures and video can be used. A simple picture can also be a form of storytelling (Sarvas and Frochlich 2011). In addition, less commonly used than pictures, audio tracks carrying simple sounds are also relevant. Work in TAG CLOUD supports these different forms of stories. The creation of advanced narratives typically requires more effort than those of pictures and audio tracks. It is necessary to study sources, collect materials, e.g. pictures, and edit media, e.g. text, audio or video. Pictures and sound tracks provide a lightweight approach to storytelling. They can be used to record an event that a person is witness of, or to highlight a detail the user is fond of.

For the user, the application module stedr is the main entry point to a cultural discovery through storytelling in TAG CLOUD. Several group interviews were organised, both with potential users and experts in various fields of cultural heritage 
in order to discuss relevant features of the storytelling module (Floch and Jiang 2015), and their feedback influenced and guided the selection and design of stedr features. Here is a brief list of feedback received and the decisions taken to develop stedr:

- Institutions have the formal responsibility for cultural places. We therefore exploit the story baseline developed by cultural institutions.

- Technology changes more rapidly than the content. The production of content is costly. Approach followed in TAG CLOUD separates between content and interaction, and there is no need to develop new stories adapted to the special needs of stedr.

- The public, in particular young people, who are under-represented among users of traditional culture, are eager users of social media. For this reason, social media is used as a support for storytelling.

- Quality and trustworthiness are essential concerns. We provide recommendations for the creation of stories, such as highlighting the importance of intellectual property rights and references.

The implementation of stedr makes use of existing platforms for storing and creating content, including some social media platforms (Floch and Jiang, Digital Heritage 2015). For instance: the digital storytelling platform for cultural stories, called Digitalt fortalt, is used for the creation and sharing of advanced narratives using different media; the social mobile picture sharing service Instagram is used for the creation and sharing of stories expressed in the form of pictures; and the social audio sharing service SoundCloud is used for the creation and sharing of stories expressed in the form of sound tracks. As far as the participatory approach is concerned, stedr supports different user roles: 'spectators' discover cultural artefacts and stories; 'critics' submit reviews to stories; 'creators' produce content, either new digital representations of cultural artefacts or stories; and 'collectors' create collections and/or organize the content into collections.

Figure 2 presents some screenshots for the application module stedr illustrating its main features. The 'map' view is the main entry point for discovery. The user can easily retrieve cultural artefact in his/her surroundings. It is however not mandatory to be close to a place to access to information. The user can browse and search on the map as usual when using Google map services. The 'story' view provides access to different kinds of stories for a cultural artefact. The 'collection' view provides access to related artefacts organised in collections. User guidelines including more screenshots can be found on the stedr blog site (stedr 2015).

Opening the public to participate in the creation of cultural stories does not mean excluding cultural institutions. Cultural institutions still play an important role. They should encourage the visitor to leave the role of observer and contribute actively, and they should educate them to produce contributions of quality. It is important to create a good baseline upon which the public can work. For instance, the institution can launch cultural themes and invite the public to contribute. Additionally, in order to lower the threshold of participation, cultural institutions 

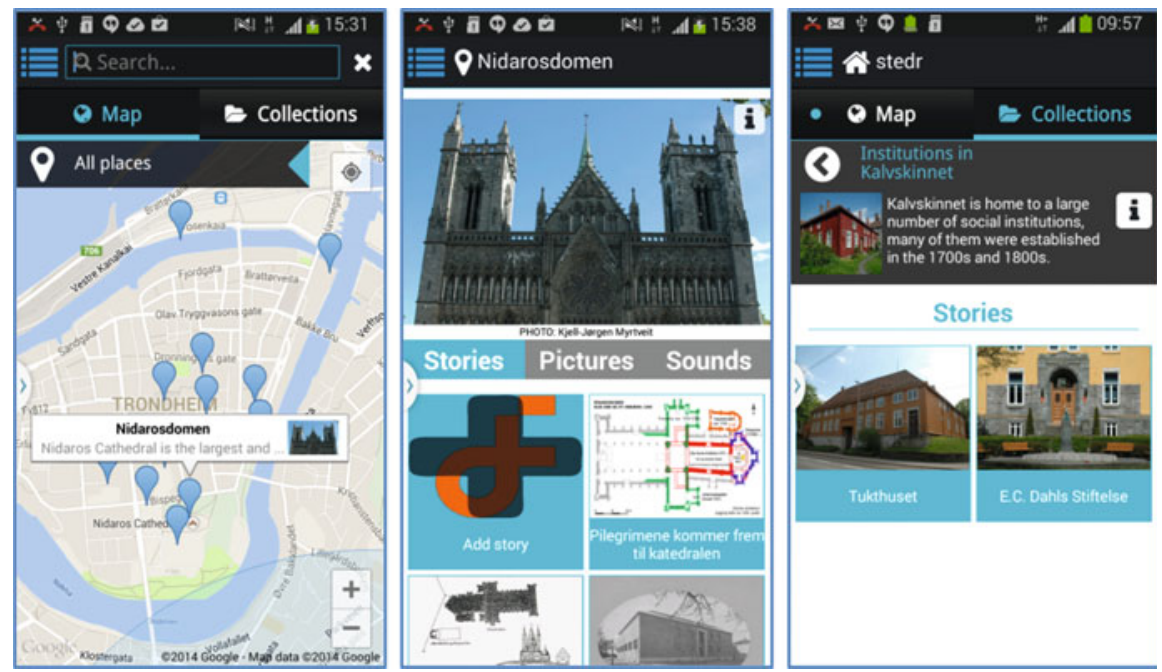

Fig. 2 Stedr screenshots. On the left, the map view, in the middle, the story view, on the right, the collection view

should make use of platforms that users are familiar with. For example, stedr exploits existing popular social media platforms like Instagram, Twitter and Flickr to ease the creation and sharing of stories (pictures), comments and collections. Using these platforms, cultural institutions can easily define hashtags when launching cultural themes. As far as quality is concerned, cultural institutions should provide guidelines to the public. This can be included as part of the digital solution done in stedr, or when more resources are available, organising workshops for the contributors.

There is great potential to utilise local citizens. Many are already actively talking about the local cultural heritage, for instance members of cultural associations. They enrich the cultural knowledge with new viewpoints, and they document parts of our culture that are yet undocumented. Many have already authored articles and books. The information is often spread verbally, not always available in a digital form, and thus difficult to retrieve. To ensure good dissemination, it is important that content is open and available through common digital infrastructures. Cultural institutions should show the way by making the content they create available through open platforms. The digital storytelling platform, Digitalt fortalt, that we exploit in stedr is such a storytelling platform managed by the Arts Council Norway. It is both open for cultural institutions and the public, thus functioning as a bridge between mediated and unmediated heritage. At the time of writing, 2400 out of more than 4000 stories currently available on Digitalt fortalt were registered by cultural institutions, indicating a fairly good portion of unmediated content.

As mentioned earlier, the quality of unmediated content requires attention. The evaluation of stedr shows that some users favour mediated content beyond unmediated content due to quality and trust concerns (Floch and Jiang 2015). At the same 
time, some other users argue that they would rather read stories written by amateurs, in particular those produced by peers or favoured by peers. It is therefore important to clearly differentiate the presentation of the mediated content from that of the unmediated content.

By using these interfaces to support the public or visitors' contributions, the stories unknown or forgotten by the cultural institutions can be well preserved and passed from generation to generation, forming a living heritage. This also contributes to social cohesion, as not just professionals from cultural institutions but also the peers can participate in storytelling, Moreover, the use of social platforms and storytelling help to enrich the cultural heritage institutions' collections, involving their audiences (including locals and visitors), and improving their audiences' communication and connection.

\section{$3 \quad$ Making the Connection Among Cultural Heritage, Places and People}

As outlined above, TAG CLOUD has explored and evaluated behavioural and social patterns in order to facilitate cultural lifelong engagement and the connection between visitors and places of cultural heritage. Overall, TAG CLOUD has worked on developing cloud-based technologies that enable cultural institutions to go beyond its spatial dimension and the one-size-fits-all approach to experience culture, moving towards the one-size-fits-one (adaptation and personalization) approach. TAG CLOUD has based its developments over the pillars of social and cultural proximity and reciprocity, and thus provides a new perspective of connecting and attracting visitors.

Overall, TAG CLOUD has been driven by the notion of cultural engagement; which is largely rooted in the recognition that lifestyles, behaviours, heritage, people and deeper knowledge of culture are all shaped by social and physical environments (people and places), and underpinned by a temporal connection. Under this rational, the TAG CLOUD project has carefully designed COOLTURA as a suite of services that allows a bidirectional and enriched relationship between people and cultural places, a better understanding of the cultural institutions and a personalized cultural experience.

Through COOLTURA, TAG CLOUD has expanded in two conceptual directions to support cultural engagement: re-escalation of the content of the cultural places and building on social connections and storytelling.

The re-escalation of the content is based in the production and consumption of the knowledge or content that is exchanged during the cultural visits. In this regard, by broadening and strengthening the cultural portfolio cultural institutions are able to create and provide a more diverse and distinctive content that is built over a wider based of knowledge, in order to better connect with the preferences of the visitor; thus more choices and alternative routes for finding out about and experiencing culture are provided. Moreover, geolocation technology allows recommendations of points of interest nearby that connect with the visitors' preferences and likes, and 
could even allow the recommendation of other cultural institutions in the visitors' immediate vicinity.

Following this flow, TAG CLOUD has developed a framework that foregrounds the benefits of adaptability and personalisation. Therefore, the COOLTURA Platform has been created as the main entry point for curators, managers and experts from cultural institutions to better communicate with people. The COOLTURA Platform allows the curation increasing quantity of digital cultural content (re-scaling the quantity and the quality of the content and its metadata) from different sources (institutions private sources and Open Source), grants the adaptation cultural resources to different perspectives and for different targeted visitors, enlists and manage the integration of Apps using emerging technologies (augmented reality, storytelling, etc.) as well as selects the devices (mobiles, tablets, smart watches, glasses, etc.) they would like to communicate through. In addition, the platform provides analytic capabilities that brings analysed information and feedback regarding the usage of the digital content and apps to cultural institutions' curators and managers, and a dashboard that allows them to know which content is consumed, by whom and through which App and device, and so creating/detecting "hot spots" for visitors, as well as creating/improving more demand oriented content and/or new apps. In addition, the analytic platform allows an evaluation and analysis of the likes, needs, preferences and trends of the users, and untapped visitors' participation by allowing an adaptive cultural experience.

The TAG CLOUD project also has explored how new insights and content can be created or used from published open data, derived from existing Europeana datasets and their combinations. This approach not only supports new versions of content but also permits third-party software developers to create new apps that enrich the TAG CLOUD platform. However, having standardised data in order to really exploit the data sets from both cultural institutions and open data sources is a very important challenge to overcome. For this purpose, the TAG CLOUD consortium decided to embed in the COOLTURA Platform harvesting tools to processes curated digital content coming from Europeana and cultural institutions. The tool maps, builds and increases the metadata structure towards the OGD (Open Government Data) metadata scheme (Open Knowledge Foundation 2015), which constitutes the base for eGovData. This tool allows COOLTURA to enable cultural institutions and third parties (software vendors, developers, intermediaries, etc.) and benefit from a content eco-system, as well as use and re-use the curated digital cultural content in contexts such as cultural engagement, tourism, creative industry or emerging ones like smart cities.

By adapting insightful content and information, TAG CLOUD empowers the building of a cultural, recreational, historical and personal perspective of the visited place. By allowing social connections and storytelling, TAG CLOUD is also able to put 'people' in the centre of cultural experiences. We have seen in the above sections that through the COOLTURA App and stedr, TAG CLOUD provides points of entrances for visitors; to co-create and digest digital cultural content in an easier, personalised, participatory and joyful way. Moreover, the TAG CLOUD consortium expects that the user-generated content (through social platforms and 
storytelling - stedr) will allow that the voice of locals and visitors to become a widely used and trusted source of information, influence the branding of the cultural institutions and making visitors active participants of the cultural experience.

Considering the road ahead and challenges in the cultural sector, COOLTURA App and Platform provide the tools and services to engage in cultural experiences; COOLTURA is underpinned by easy, fun and personalised access to the digital heritage trusted knowledge eco-system (and to the stories to be told) from the collections, monuments or areas (cities, neighbourhoods, etc.) that mark the time and place of the what, where and how we have lived our lives (culture), what has happened in the different parts of the Earth, or what, where and how other species have lived.

\section{The Value of Connecting People and Places}

From an empirical and qualitative evaluation performed during the late stage of TAG CLOUD project for exploitation (TAG CLOUD 2015), cultural institutions reported that the way COOLTURA App and Platform can create value is by linking the actions that result from its usage with the policies, visions and missions of the site; and thus connect with the mind and emotion of the user.

As in the case of the Alhambra, many small and large cultural institutions, organisations, monuments and cities reported that they were willing and ready to adopt emerging technologies related with personalisation and customised services, and new ways to deliver digital cultural content and resources. However, coupled with this process, the cultural institutions see the need to adopt organisational processes that link to their policies and core mission, in order to really get full alignment of curatorial, marketing and educational cultural resources and capture the attention of today's visitors.

In this regard we can see a large cultural monument such as it is the Monumental Complex of Alhambra and Generalife (Granada, Spain). The Alhambra, as a case for exploiting the aims of the TAG CLOUD project and linking COOLTURA with their policies of being a safe, clean, well maintained, serviced and restored distinctive cultural place where people not only visit as a 'cultural must' but also enjoy visiting. The Alhambra and Generalife Monumental Complex, is not only about the historical palatial cities and the Generalife, it comprises and promotes other cultural interventions such as events (e.g. concerts and exhibits from local artists), an archive, a library, nearby hostelry and food, as well as a green and sustainable areas with gardens and a developed green environment, where experts, lecturers or students give special botanical tours. Moreover, other cultural places in Granada and local green public spaces play an important part in the development and motivation to create the Alhambra and its surroundings as a pleasant environment for and by locals, businesses and visitors.

For the Alhambra, the aim of these cultural interventions, what we call cultural 'placemaking', is that people and visitors can look at the Alhambra as a cultural entity embodied in the culture of Andalusia, and not simply as set of individual 
cultural buildings. The use of all these as cultural interventions lets people look at the Monumental Complex of the Alhambra in a different way; one that aims at improving the connection with the diversity and quality of the cultural values of visitors, locals and businesses. On this subject, through the curation of easy digestible and personalise content, as well as initiatives such as storytelling, COOLTURA allows new cultural values to be given to the Alhambra through new narratives that make people look at the Alhambra from different perspectives. So the 'place' and its culture, can gain in value and appreciation when COOLTURA's new layout is provided to people and visitors: a place where they can write and consume stories, good recommendations and cultural content. This new layer aims at enhancing the appreciation of places and its culture by making places closer to people, people closer to places, as well as changing the way they feel about places and places connect with people. It is about creating and managing the digital cultural content to support a lively cultural place and prove enthusiasm about it that reaches multiple identities with families, visitors and communities that enjoy and share different cultures.

Contrary to large monuments such as the Monumental Complex of Alhambra, cultural institutions (e.g. museums and monuments) in small towns and villages receive often less attention than more well-known cultural institutions in large cities. Often, few resources are available to create digital content about these institutions, and to develop and maintain a digital infrastructure for storing and disseminating that content. TAG CLOUD can address these challenges. The common digital COOLTURA Platform supports the recommendation of cultural institutions. Less known sites will be recommended if they match the preferences of the users. The COOLTURA Platform harvests information from common cultural digital infrastructures, e.g. Europeana, and can be extended for harvesting information from other common or proprietary infrastructures. For instance, COOLTURA Platform harvests content from the Norwegian storytelling platform Digitalt fortalt that any cultural institution in Norway can use to create and share cultural stories. Furthermore, it supports a participatory approach and lets the public contribute with contents, both comments about sites and cultural stories. In particular, less known places can be promoted using the social media plugins of COOLTURA and the TAG CLOUD storytelling component stedr. No cultural site or institution is too small for TAG CLOUD. An example is the case of the small island Rødøya in northern Norway. Rødøya is a little gem on the coast of Helgeland close to the polar circle. The small island with 200 inhabitants receives 25,000 visitors every year, mainly in the summer time. The island has been a major church centre and trading place for several hundred years. The project "Opp i dagen" (i.e. "bringing to light") has gathered experts from different culture and nature disciplines (e.g. history, archaeology and geology) in order to document the island's cultural heritage. The result is a book and a set of information signs. They exploit stedr in order to support digital interaction with their visitors. As the content was already available in a digital form, little effort was needed to make digital stories about Rødøya available through stedr. The new cultural offer was launched in Rødøya at the end of May, 2015 (Floch, TAG CLOUD 2015) (Ranablad 2015). 
By embracing these challenges, the TAG CLOUD project is deploying COOLTURA to support an invigorating transformation of cultural places (large and small), making places and cultural information accessible, adaptable and personalized to people through emerging cloud-based technologies; and thus bridging a bidirectional connection between people and places, at the heart of an pro-active public realm. Moreover, through COOLTURA, the TAG CLOUD project has tackled the idea that places are "frozen in time" by re-scaling the exchange of content and knowledge in an adaptive manner, while building and enriching places with social, cultural and personal perspectives.

Acknowledgment The work presented in this manuscript has been partially funded by the EC FP7 project TAG CLOUD (Technologies lead to Adaptability \& lifelong enGagement with culture throughout the CLOUD); http://www.tagcloudproject.eu, Grant Agreement No. 600924.

Open Access This chapter is distributed under the terms of the Creative Commons AttributionNoncommercial 2.5 License (http://creativecommons.org/licenses/by-nc/2.5/) which permits any noncommercial use, distribution, and reproduction in any medium, provided the original author(s) and source are credited.

The images or other third party material in this chapter are included in the work's Creative Commons license, unless indicated otherwise in the credit line; if such material is not included in the work's Creative Commons license and the respective action is not permitted by statutory regulation, users will need to obtain permission from the license holder to duplicate, adapt or reproduce the material.

\section{References}

Ardissono, L., Kuflik, T., \& Petrelli, D. (2012). Personalization in cultural heritage: The road travelled and the one ahead. User Modeling and User-Adapted Interaction, 22(1-2), 73-99.

Bernstein, S. (2008). Where Do We Go From Here? Continuing with Web 2.0 at the Brooklyn Museum. In J. Trant \& D. Bearman (Eds.), Museums and the Web 2008. Proceedings, Toronto: Archives \& Museum Informatics. Retrieved December 7, 2015, from http://www.archimuse. com/mw2008/papers/bernstein/bernsteinhtml.

Borowiecki, K. J., \& Navarrete, T. (2015). Digitization of heritage collections as indicator of innovation. Retrieved December 7, 2015, from https://ideas.repec.org/p/hhs/sdueko/2015_014. html.

Borowiecki, K. J., \& Navarrete, T. (2016). Digitization of heritage collections as indicator of innovation. Economics of Innovation and New Technology, forthcoming. Retrieved March 14, 2016, from https://ideas.repec.org/p/hhs/sdueko/2015_014.html.

European Commission. (2014). Cultural heritage. Digitisation, online accessibility and digital preservation. Report on the Implementation of Commission Recommendation 2011/711/EU. Progress report 2011-2013. Retrieved December 7, 2015, from ec.europa.eu/newsroom/dae/ document.cfm?doc_id=9745

European Commission. (2007, May 10). Press releases of the European Commission. Retrieved December 7, 2015, from http://europa.eu/rapid/press-release_IP-07-646_en.htm

European Commission. (2014, May 20). Press releases of the European Commission. Retrieved December 7, 2015, from http://ec.europa.eu/culture/news/2014/20140602-heritage_en.htm.

"Europeana" platform. Retrieved December 7, 2015, from http://www.europeana.eu/ 
Falk, J. H. (2009). Identity and the museum visitor experience. Walnut Creek, CA: Left Coast Press.

Floch, J. (2015, June 1). News post at TAG CLOUD website. Retrieved December 7, 2015, from http://www.tagcloudproject.eu/?p=1520

Floch, J., \& Jiang, S. (2015, September 22-24) Digital cultural experiences: A step towards understanding the public needs. Paper presented at the 6th International Workshop on HumanComputer Interaction, Tourism and Cultural Heritage-HCITOCH, Ravenna, Italy.

Floch, J., \& Jiang, S. (2015) One place, many stories - Digital storytelling for cultural heritage discovery in the landscape. Paper presented at the Digital Heritage International Congress 2015, Granada, Spain, September 28-October 2, 2015.

Open Knowledge Foundation. Retrieved December 7, 2015, from https://okfn.org/

Ranablad. (2015, June 1), blog post, Skatter opp i dagen på Rødøya. Retrieved December 7, 2015, from http://www.ranablad.no/historie/rodoy/nord-norges-storste-myntfunn-fra-middelalderenble-gjort-pa-hamnoy-ved-rodoya-for-over-100-ar-siden/s/5-42-62144

Saldaña, I., Celaya, J., Merin, C., Gonzalez, C., Corbo, A., Portell, G., et al. (2013). Museums in the digital age. Retrieved 7, 2015, from http://www.dosdoce.com/upload/ficheros/noticias/ 201305/museums_in_the_digital_age_a_dosdoce_survey.pdf

Sarvas, R., \& Frohlich, D. M. (2011). From snapshots to social media-the changing picture of domestic photography: The changing picture of domestic photography. London: Springer Science \& Business Media.

Simon, N. (2010) The participatory museum. Museum 2.0.

Special Eurobarometer 399. (2013, November). Cultural access and participation. Retrieved December 7, 2015, from http://ec.europa.eu/public_opinion/archives/ebs/ebs_399_en.pdf

"stedr" blog site. Retrieved December 7, 2015, from http://stedr.blogspot.no

TAG CLOUD project, CN. 600924, 7th Framework Programme, ICT for access to cultural resources, February, 2013; in-depth interviews for COOLTURA Platform exploitation.

Vassileva, J. (2012). Motivating participation in social computing applications: a user modeling perspective. User Modeling and User-Adapted Interaction, 22(1-2), 177-201.

Wilkening, S., \& Chung, J. (2009). Life stages of the museum visitor: Building engagement over a lifetime. Washington, DC: American Association of Museums Press. 